\title{
Molecular Diagnosis of Toxoplasma gondii in Aborted Women
}

\author{
Fatemeh Sadat Ghasemi ${ }^{1}$; Sima Rasti ${ }^{1, *}$; Mojgan Bandehpour ${ }^{2,3}$; Bahram Kazemi ${ }^{2,3}$; Ahmad \\ Piroozmand ${ }^{4}$; Gholam Abbas Mousavi $^{5}$ \\ ${ }^{1}$ Department of Parasitology, Faculty of Medicine, Kashan University of Medical Sciences, Kashan, IR Iran \\ ${ }^{2}$ Cellular and Molecular Biology Research Center, Shahid Beheshti University of Medical Sciences, Tehran, IR Iran \\ 3 Biotechnology Department, Faculty of Medicine, Shahid Beheshti University of Medical Sciences, Tehran, IR Iran \\ Biotechnology Department, Faculty of Medicine, Shahid Beheshti University of Medical Sciences, Tehran, IR Iran
${ }^{4}$ Department of Microbiology and Immunology, Faculty of Medicine, Kashan University of Medical Sciences, Kashan, IR Iran \\ 5 Department of Microbiology and Immunology, Faculty of Medicine, Kashan University of Medical Sciences, Kashan, IR ir
${ }_{\text {Department }}$ Statistics and Public Health, Faculty of Health, Kashan University of Medical Sciences, Kashan, IR Iran \\ *Corresponding author: Sima Rasti, Department of Parasitology, Faculty of Medicine, Kashan University of Medical Sciences, Kashan IR Iran. Tel: +98-9132611568, Fax:+98-3615551112, \\ E-mail:Rasti_s@yahoo.com
}

Received: November 2, 2013; Revised: December 25, 2013; Accepted: January 14, 2014

Keywords:Toxoplasma gondii; Polymerase Chain Reaction; Placenta

\section{Dear Editor,}

Infection with Toxoplasma gondii during pregnancy may cause severe damages to the fetus including spontaneous abortion, stillbirth and neurological signs. Its severity is gestational time-dependent (1). According to previous studies, maternal infection in the first and second trimester may be resulted in prenatal death or stillbirth in 5\% and $2 \%$ of the cases, respectively $(1,2)$. The major diagnostic assays for toxoplasmosis are serological methods despite many limitations (3). Molecular method have been developed to improve the diagnosis of congenital toxoplasmosis (3). Polymerase Chain Reaction (PCR)-based assay to amplify a 200-300 fold reprtitive 529-base pair (bp) DNA region of Toxoplasma has a higher sensitivity than conventional serological tests (4). Development of an accurate, sensitive and rapid method for the detection and identification of $T$. gondii is important in both diagnosis and treatment (5).

The objective of this study was to detect T. gondii DNA in placenta by PCR among aborted women and those with normal delivery. This case-control study carried out on 110 women, whose pregnancies were spontaneously terminated at less than 20 weeks that considered as case group and the same sample group of pregnant women with gestational age of 38-39 weeks which had normal delivery was included in the control group, hospitalized to hospitals (Akbar abadi, Mirza kochak Khan and Hedayat) in Tehran, Iran from September 2012 to August 2013. A questionnaire including demographic and risk factors along with symptoms were used. DNA of $0.5 \mathrm{~g}$ of placenta samples were extracted using DNA extraction kit, according to the manufacturer's instructions (Bioneer, Korea). PCR was carried out to amplify the $400 \mathrm{bp}$ segments of
T. gondii by primer G529 with annealing temperature of $48^{\circ} \mathrm{C}$ for $30 \mathrm{sec}$ (6). Forward 5'-TTT TGA CTC GGG CCC AGC 3'; Reverse; 5'-GTC CAA GCC TCC GAC TCT 3'.

T. gondii RH strains and DDW were used as positive and negative controls, respectively. PCR product was electrophoresed on a 1.5\% agarose gel. A 400 bp band, designated as positive PCR. All the data were analyzed by SPSS version 13.5 using Chi squared and Fisher's exact tests. This study was approved by the ethical committee of Kashan University of Medical Sciences, Iran. A total of 220 pregnant women with an age range of 17-46 years were divided in two case and control groups. All the demographic and risk factors along with symptoms except the history of abortion in the case and control groups were similar. In this novel study, the G529 gene (a fragment of $T$. gondii DNA) was amplified by PCR from samples collected from 7 (6.4 \%) of the aborted women and stillbirth and $2(1.8 \%)$ of the control group $(\mathrm{PV}=0.17)$ (Table 1 and Figure 1$)$.

Table 1. The Prevalence of T. gondii PCR Positive Results Among Aborted Women and Those With Normal Delivery ${ }^{\mathrm{a}, \mathrm{b}}$

\begin{tabular}{lcc}
\hline Group & Case & Control \\
\hline PCR & & \\
Positive & $7(6.4)$ & $2(1.8)$ \\
Negative & $103(93.6)$ & $108(98.2)$ \\
Total & 110 & 110 \\
P.V & \multicolumn{2}{c}{0.17} \\
OR & \multicolumn{2}{c}{3.67} \\
CI & \multicolumn{3}{c}{$0.745-18.07$} \\
\hline
\end{tabular}

a Abbreviations: PCR, Polymerase Chain Reaction; P.V, P value; OR, Odds Ratio; CI, Confidence Interval.

$\mathrm{b}$ values are present as No. (\%). 
Figure 1. The PCR Product T. gondii From Aborted Placenta

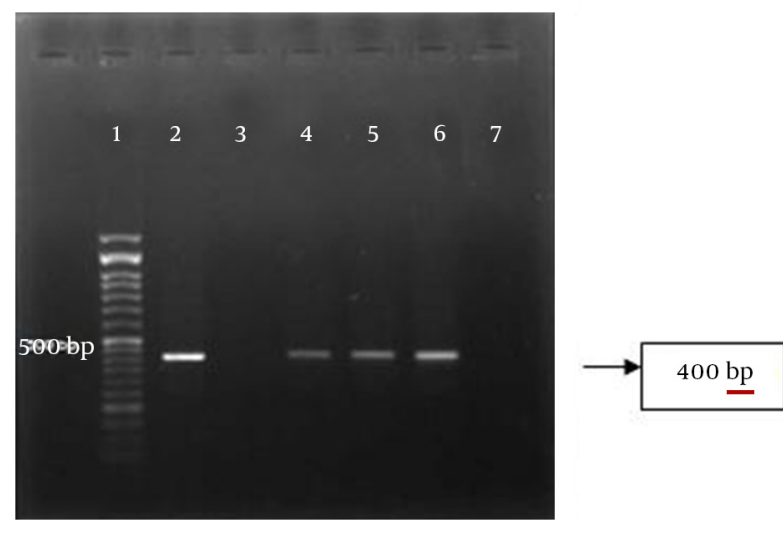

Lane 1, DNA ladder $50 \mathrm{bp}$, lane 2 and 3, positive and negative controls; lane 4-6, positive samples, lane 7, negative sample.

The rate of $T$. gondii PCR positive results among the women with spontaneous abortion and stillbirth were 6 (85.7\%) and 1 (14.3\%), respectively. The highest prevalence of $T$. gondii infection was among the age group of 20-29 years and undergraduate and housekeepers women. Based on false negativity in serological data, PCR using the placental tissue is useful for the rapid and accurate diagnosis of congenital toxoplasmosis $(4,6)$. In our study, the rate of T. gondii infection using PCR and G529 primer was 3.67 times higher in the aborted placenta compared to those with normal delivery, but the difference was not significant $(\mathrm{P}=0.17)$. Asgari et al. in Shiraz, Iran reported that $14.4 \%$ of paraffin-embedded blocks of aborted placenta were shown PCR-positive results (7), which was higher than the results of our study. The type and size of the sample may affect the outcome of the study. According the results of Fajrk et al. seven out of $35(20 \%)$ fetal death had positive PCR results (8).

Time of abortion or fetal death, PCR set up and primer type can affect the results of PCR. Nowakowska et al. showed two out of four prenatal samples of placenta and one out of 14 samples with primary infection pregnancy diagnosed by serology, were T. gondii DNA positive (9). The results of this study showed that in spite of higher prevalence of T. gondii in aborted women compared to those with normal delivery, there is no significant relationship between toxoplasmosis and abortion, but T. gondii infection could be considered as risk factor of abortion and that molecular method, especially PCR has important role for detecting T. gondii DNA from placenta samples.

\section{Acknowledgements}

The authors thank the vice chancellor of Research Deputy of Kashan University of Medical Sciences for his financial support (No. 9153). The authors acknowledged wholehearted cooperation of the Maternity Clinics in Tehran, Iran and Dr. Falahian and Dr. Hoosien Hooshyar.

\section{Funding/Support}

This study has been financially supported by the Grant No 9153, Kashan University of Medical Sciences.

\section{References}

1. Montoya JG, Liesenfeld O. Toxoplasmosis. Lancet. 2004;363(9425):1965-76.

2. Dubey JP. Toxoplasmosis. In: Collier L, Sussman M editors. Toply and Wilson's Microbiology and Microbial infection.. USA: Arnold Copublished; 2005. p. 303.

3. Pignanelli S. Laboratory diagnosis of Toxoplasma gondii infection with direct and indirect diagnostic techniques. Indian $J$ Pathol Microbiol. 2011;54(4):786-9.

4. Abdul-Ghani R. Polymerase chain reaction in the diagnosis of congenital toxoplasmosis: more than two decades of development and evaluation. Parasitol Res. 2011;108(3):505-12.

5. Slawska H, Pendzich J, Czuba B, MazurekU, Gola J, Wilczok T, et al [Detection of Toxoplasma gondii DNA by PCR in mother's blood, amniotic fluid and child's blood in selected cases of pathological pregnancy]. Wiad Parazytol. 2001;47 Suppl 1:99-105.

6. Rasti S, Behrashi M, Kazemi B, Fatahian A, Mousavi G, Namak chian M. Diagnosis of congenital toxoplasmosis by polymerase chain reaction. Indian J Med Microbiol. 2012;30(2):251.

7. Asgari Q, Fekri M, Monabati A, Kalantary M, Mohammadpour I, Motazedian MH, et al. Molecular Genotyping of Toxoplasma gondii in Human Spontaneous Aborted Fetuses in Shiraz, Southern Iran. Iran J Public Health. 2013;42(6):620-5.

8. Fajrk H. [Evaluation of fetal death caused by Toxoplasma PCR method]. Shahid Beheshti Univ Med Sci.1996.

9. Nowakowska D, Golab E, Czichos E, Krekora M, Wilczynski J. [Detection of Toxoplasma gondii in human placenta by PCR and placental histologic findings]. Wiad Parazytol. 2002;48(3):301-9. 